\title{
Association between autonomic dysfunction and olfactory dysfunction in Parkinson's disease in southern Chinese
}

\author{
Xin-Yi Wang ${ }^{\dagger}$, Ying-Ying Han ${ }^{\dagger}$, Gang Li and Bei Zhang ${ }^{*}$
}

\begin{abstract}
Background: The aim was to investigate the autonomic dysfunction between Parkinson's disease (PD) patients with olfactory dysfunction and PD patients without olfactory dysfunction in southern Chinese population.

Methods: Fifty-six PD patients with olfactory dysfunction and 44 patients without olfactory dysfunction were included. All patients were evaluated by Sniffin' sticks (SS-16), scales for outcomes in Parkinson's disease-autonomic questionnaire, Hamilton anxiety rating scale and Hamilton depression rating scale

Results: The score of subpart of gastrointestinal symptoms and subpart of urinary symptoms were different in two groups (gastrointestinal symptoms: $p$ value: 0.024 ; urinary symptoms: $p$ value: 0.008 ). As for each question items, questions 2, 8, 10, 11, 13, 14 were correlated with SS-16 scores (Question 2: $p$ value: 0.013; question 6: $p$ value: 0. 006; question 8: $p$ value: 0.025; question 10: $p$ value: 0.005 ; question 11: $p$ value: 0.022 ; question 13: $p$ value: $<0.001$; question 14: $p$ value: 0.038 ). Question 10 and 14 were associated with olfactory dysfunction after adjusting disease duration and gender (Question 10: $p$ value: 0.011, OR: 3.91; Question 14: $p$ value: 0.027, OR: 3.27).
\end{abstract}

Conclusions: Gastrointestinal, urinary and a part of cardiovascular symptoms of SCOPA-AUT were associated with olfactory dysfunction in PD patients.

Keywords: Parkinson's disease, Olfactory dysfunction, Autonomic dysfunction

\section{Background}

Parkinson's disease (PD) is a progressive movement disorder characterized by bradykinesia, rigidity and resting tremor [1]. The pathological characteristics of PD are the loss of dopaminergic neurons in the substantia nigra and abnormal $\alpha$-synuclein aggregation [2]. We can divide PD symptoms into two parts, motor symptoms, such as rigidity, and non-motor symptoms such as constipation and olfactory dysfunction [3].

Olfactory dysfunction is a symptom and biomarker in PD $[4,5]$. It is also a risk marker of prodromal PD [6]. Now it is taken more seriously since it is a part of peripheral symptoms. Autonomic dysfunction is also common in PD. Its symptoms can vary with different subsystems, such as constipation and cardiovascular dysautonomia [7-9]. Constipation is a risk marker of prodromal PD [6]. Both olfactory dysfunction and several autonomic dysfunctions such as constipation present prodromal biomarkers for PD with peripheral origins. But rare studies focused on their relationships in PD patients. The differences of autonomic dysfunction of PD patients with olfactory dysfunction and without olfactory dysfunction are rarely discussed. It is useful to perform early and comprehensive management in PD patients if we know better the association between olfactory function and autonomic function. In this study, we try to discover differences of several autonomic symptoms between PD patients with olfactory dysfunction and patients without olfactory dysfunction in southern Chinese PD population.

\footnotetext{
* Correspondence: zhangbei0227@163.com

${ }^{+}$Xin-Yi Wang and Ying-Ying Han contributed equally to this work.

Department of Neurology, East Hospital, Tongji University School of

Medicine, 1800 Yuntai Road, Shanghai 200123, China
}

(c) The Author(s). 2019 Open Access This article is distributed under the terms of the Creative Commons Attribution 4.0 International License (http://creativecommons.org/licenses/by/4.0/), which permits unrestricted use, distribution, and reproduction in any medium, provided you give appropriate credit to the original author(s) and the source, provide a link to the Creative Commons license, and indicate if changes were made. The Creative Commons Public Domain Dedication waiver (http://creativecommons.org/publicdomain/zero/1.0/) applies to the data made available in this article, unless otherwise stated. 


\section{Methods}

\section{Study population}

PD was collected from outpatients clinic of shanghai East hospital and diagnosed by movement disorder specialists based on diagnostic criteria brought up by movement disorders society (MDS) [10]. As for PD patients, Hoehn-Yahr staging and their disease duration were recorded. We excluded common secondary causes, such as inflammatory, drug-induced, vascular and toxin-induced parkinsonism. Parkinsonism with other neurodegenerative diseases, such as progressive supranuclear palsy, Wilson's disease, cerebral-basal degeneration and multiple system atrophy was also excluded. Patients with history of operations of nose, chronic rhinitis, recent upper respiratory tract infection and smoking were also excluded. We also excluded patients with anxiety or depression using Hamilton anxiety rating scale and Hamilton depression rating scale. Patients with cognitive dysfunction assessed by Chinese version Mini-mental State Examination and Montreal Cognitive Assessment - Beijing version were ruled out. All participants signed consent forms. This study was approved by the ethic committee of shanghai East hospital.

\section{Olfactory tests and questionnaire evaluation}

Olfactory test (Sniffin' Sticks, SS-16) was performed to assess olfactory function through 16-item odor identification [11]. Since the threshold of SS-16 in Chinese population is $7.22 \pm 1.75$, we divided PD patients into two groups, PD patients with olfactory dysfunction (score of SS-16 <5) AND PD patients without olfactory dysfunction (score of SS-16> 9) [12]. Chinese version of Scales for Outcomes in Parkinson's Disease-Autonomic questionnaire (SCOPA-AUT) was used to assess autonomic symptoms [13]. Hamilton anxiety rating scale and Hamilton depression rating scale were used to assess anxiety and depression. Researchers were received strict training of these scales before assessing PD patients.

\section{Statistics}

$\mathrm{R}$ (version 3.5.0), stats package (version 3.5.0) was used to perform statistical analysis. $t$ test was used to compare differences of age, disease duration and SS-16 scores between PD patients with olfactory dysfunction and patients without olfactory dysfunction. SCOPA-AUT and its subparts was assessed by multivariate regression adjusted by disease duration, Hoehn - Yahr staging, age and gender. Chi-square test was used in comparing differences of gender and $2 \times 2$ tables in individuals of SCOPA-AUT between two groups. Linear correlation was used to assess the correlation between the score of individuals of SCOPA-AUT and SS-16 scores. Logistic regression analysis was performed with odds ratios (OR) and 95\% confidence intervals (CI) calculated. Disease duration, age and gender were also adjusted. A level of $P<.05$ was regarded as statistically significant.

\section{Results}

In total, 56 PD patients with olfactory dysfunction and 44 PD patients without olfactory dysfunction were included in our study. There was no difference of age, gender and body mass index between PD patients with olfactory dysfunction and PD patients without olfactory dysfunction. There was no statistical difference of Hoehn - Yahr staging and disease duration between PD patients with olfactory dysfunction and PD patients without olfactory dysfunction. Of PD patients with olfactory dysfunction, the average age when they were tested was 68.29 years old and the average disease duration was 7.86 years. There was a statistical significant difference of the score of SS-16 between two groups (PD patients with olfactory dysfunction: $1.55 \pm 1.04$; PD patients without olfactory dysfunction: $11.93 \pm 1.11, p$ value: $<0.001$ ). However, we found statistical differences of total score of SCOPA-AUT between two groups (PD patients with olfactory dysfunction: $14.73 \pm 9.05$; $\mathrm{PD}$ patients without olfactory dysfunction: $9.75 \pm 7.37, \mathrm{p}$ value: 0.008$)$. As for the score of subpart of gastrointestinal symptoms and subpart of urinary symptoms in SCOPA-AUT, there were statistical differences between two groups (Subpart of gastrointestinal symptoms: PD patients with olfactory dysfunction: $5.00 \pm$ 4.11, PD patients without olfactory dysfunction: 3.07 $\pm 3.00, p$ value: 0.024; Subpart of urinary symptoms: PD patients with olfactory dysfunction: $5.38 \pm 4.49$, PD patients without olfactory dysfunction: $2.89 \pm 3.80$, $p$ value: 0.008 ) (Table 1 ).

As for each question items, the scores of question 10 "feeling that after passing urine your bladder was not completely empty" and question 14 "lightheaded when standing up" were correlated with SS-16 scores (question 10: $p$ value: 0.005 ; question $14: p$ value: 0.038$)$. The symptoms of question 10 and question 14 were also associated with olfactory dysfunction, with or without adjusting disease duration and gender (question 10: $p$ value: 0.006 , OR: 4.10 , before adjustion; $p$ value: 0.011, OR: 3.91, after adjustion; question 14: $p$ value: 0.030, OR: 2.94, before adjustion; p value: 0.027 , OR: 3.27, after adjustion). As for the correlation with SS-16 scores, the score of question 2 "saliva dribbled out of your mouth", question 6 "strain hard to pass stools", question 8 "difficulty retaining urine", question 11 "the stream of urine is weak" and question 13 "have to pass urine at night" were presented these correlations (Question 2: $p$ value: 0.013; question 6: $p$ value: 0.006 ; question $8: p$ value: 0.025 ; question $11: p$ value: 0.022 ; question 13: $p$ value: <0.001). Besides, the symptom that question 6 described was associated with olfactory dysfunction before adjusting disease duration and gender (question 6: $p$ value: 0.030 , OR: 2.45 , before adjustion) (Table 2). 
Table 1 Demographic data of PD patients with olfactory dysfunction and PD patients without olfactory dysfunction

\begin{tabular}{|c|c|c|c|}
\hline & $\begin{array}{l}\text { PD patients with olfactory dysfunction } \\
(N=56)\end{array}$ & $\begin{array}{l}\text { PD patients without olfactory dysfunction } \\
(N=44)\end{array}$ & $p$ value \\
\hline Age, mean (SD) & $68.29(7.64)$ & $67.09(8.35)$ & 0.463 \\
\hline Gender, female, N (\%) & $29(51.79)$ & $19(43.18)$ & 0.393 \\
\hline Body mass index, mean (SD) & $22.19(2.11)$ & $22.42(2.46)$ & 0.631 \\
\hline Duration, mean (SD) & $7.86(4.40)$ & $6.86(5.29)$ & 0.319 \\
\hline \multicolumn{4}{|l|}{ Hoehn - Yahr Staging, N (\%) } \\
\hline 1.0 & $9(16.07)$ & $14(31.82)$ & \multirow[t]{7}{*}{0.242} \\
\hline 1.5 & $7(12.50)$ & $7(15.91)$ & \\
\hline 2.0 & $18(32.14)$ & $11(25.00)$ & \\
\hline 2.5 & $13(23.21)$ & $5(11.36)$ & \\
\hline 3.0 & $5(8.93)$ & $6(13.64)$ & \\
\hline 4.0 & $4(7.14)$ & $1(2.27)$ & \\
\hline 5.0 & $0(0)$ & $0(0)$ & \\
\hline SS-16, mean (SD) & $2.54(1.56)$ & $11.93(1.11)$ & $<0.001$ \\
\hline HARS, mean (SD) & $1.55(1.04)$ & $1.39(1.24)$ & 0.476 \\
\hline HDRS, mean (SD) & $1.52(1.19)$ & $1.32(1.20)$ & 0.409 \\
\hline SCOPA - AUT, mean $(S D)^{*}$ & $14.73(9.05)$ & $9.75(7.37)$ & 0.008 \\
\hline Gastrointestinal symptoms* & $5.00(4.11)$ & $3.07(3.00)$ & 0.024 \\
\hline Urinary symptoms* & $5.38(4.49)$ & $2.89(3.80)$ & 0.008 \\
\hline Cardiovascular symptoms* & $0.70(1.11)$ & $0.32(0.86)$ & 0.076 \\
\hline Skin symptoms* & $2.70(2.48)$ & $2.66(2.81)$ & 0.793 \\
\hline Sexual symptoms* & $0.50(1.54)$ & $0.55(1.45)$ & 0.804 \\
\hline Drug usage* & $0.46(0.66)$ & $0.50(1.68)$ & 0.783 \\
\hline
\end{tabular}

HARS Hamilton Anxiety Rating Scale, HDRS Hamilton Depression Rating Scale, PD Parkinson's Disease, SD Standard Deviation, SCOPA-AUT Scales for Outcomes in Parkinson's Disease-Autonomic questionnaire

* adjusted age, gender, disease duration and Hoehn-Yahr staging

\section{Discussion}

Our study found that differences of urinary, cardiovascular and gastrointestinal symptoms between PD patients with olfactory dysfunction and PD patients without olfactory dysfunction. To our knowledge, this is the first study to demonstrate these phenomena in PD patients in southern Chinese population.

In PD patients, constipation is the most common gastrointestinal problems. Olfactory dysfunction and constipation are both prodromal PD symptoms. There were several studies proved their associations $[14,15]$. In undiagnosed individuals, hyposmia is associated with other non-motor PD symptoms, such as constipation [16]. Anosmia and constipation could progress with the primary disease process [17]. However, several researches may indicate that olfactory dysfunction and constipation may do not share the same pathogenesis. No differentially abundant gut microbes which were observed between PD and healthy individuals were replicated in nasal microbiome research [18].

The relationship between olfactory dysfunction and constipation in PD patients may not share same microbes via gut-brain axis. Besides, dopamine transporter activities are different between constipation and hyposmia in PD patients. Constipation was not associated with dopamine transporter (DAT) pathology in early drug-naïve PD patients with the help of DAT single-photon emission computed tomography (SPECT) [19]. Interestingly, idiopathic hyposmia patients who have highly chance to develop into PD were associated with the DAT pathology [20]. In PD patients, hyposmia was also correlated with striatal dopamine innervation shown by DAT binding [21]. The further relationship between olfactory dysfunction and constipation is warranted to be discovered.

Hyposmia is associated with cardiovascular dysfunction. Smell scores were associated with heart rate variability [15]. Smell scores were also associated with the increase of blood pressure of norepinephrine and dobutamine infusion tests and the uptake of cardiac ${ }^{123}$ I-metaiodobenzylguanidine (MIBG) [22]. Smell scores were also correlated with cardiac sympathetic degeneration presenting with early and delay heart to mediastinum ratio and the washout rate with early PD patients 
Table 2 Association between individual items of SCOPA-AUT and olfactory dysfunction

\begin{tabular}{|c|c|c|c|c|c|c|c|}
\hline \multirow[t]{2}{*}{ Questions } & \multirow[t]{2}{*}{ Correlations with SS-16 ${ }^{a}$} & \multicolumn{3}{|c|}{$\begin{array}{l}\text { Correlations with } \\
\text { individual }^{\mathrm{b}}\end{array}$} & \multicolumn{3}{|c|}{$\begin{array}{l}\text { Correlations with individual } \\
\text { (adjusted) }^{b, c}\end{array}$} \\
\hline & & $p$ value & OR & $95 \% \mathrm{Cl}$ & $p$ value & OR & $95 \% \mathrm{Cl}$ \\
\hline Question 1 "difficulty swallowing or have you choked" & 0.306 & 0.950 & 1.05 & $(0.22,5.58)$ & 0.954 & 1.05 & $(0.21,5.96)$ \\
\hline Question 2 "saliva dribbled out of your mouth" & 0.013 & 0.285 & 1.55 & $(0.70,3.46)$ & 0.439 & 1.16 & $(0.60,3.18)$ \\
\hline Question 3 "food become stuck in your throat" & 0.078 & 0.273 & 2.52 & $(0.55,17.82)$ & 0.153 & 4.92 & $(0.93,70.09)$ \\
\hline Question 4 "full very quickly during a meal" & 0.144 & 0.230 & 1.91 & $(0.68,5.91)$ & 0.264 & 1.87 & $(0.64,6.04)$ \\
\hline Question 5 "constipation" & 0.178 & 0.119 & 1.89 & $(0.85,4.25)$ & 0.284 & 1.62 & $(6.69,3.97)$ \\
\hline Question 6 "strain hard to pass stools" & 0.006 & 0.030 & 2.45 & $(1.10,5.60)$ & 0.066 & 2.24 & $(0.96,5.35)$ \\
\hline Question 7 "involuntary loss of stools" & 0.194 & 0.996 & - & - & 0.996 & - & - \\
\hline Question 8 "difficulty retaining urine" & 0.025 & 0.405 & 1.43 & $(0.62,3.38)$ & 0.521 & 1.33 & $(0.56,3.18)$ \\
\hline Question 9 "involuntary loss of urine" & 0.705 & 0.182 & 0.49 & $(0.16,1.39)$ & 0.091 & 0.37 & $(0.11,1.14)$ \\
\hline $\begin{array}{l}\text { Question } 10 \text { "feeling that after passing urine your bladder was } \\
\text { not completely empty" }\end{array}$ & 0.005 & 0.006 & 4.10 & $(1.56,12.21)$ & 0.011 & 3.91 & $(1.45,12.00)$ \\
\hline Question 11 "the stream of urine is weak" & 0.022 & 0.318 & 1.73 & $(0.61,5.37)$ & 0.286 & 1.83 & $(0.62,5.95)$ \\
\hline Question 12 "pass urine again within $2 \mathrm{~h}$ of the previous time" & 0.064 & 0.915 & 0.95 & $(0.40,2.30)$ & 0.682 & 0.82 & $(0.31,2.14)$ \\
\hline Question 13 "have to pass urine at night" & $<0.001$ & 0.115 & 1.93 & $(0.86,4.40)$ & 0.144 & 1.85 & $(0.81,4.27)$ \\
\hline Question 14 "lightheaded when standing up" & 0.038 & 0.030 & 2.94 & $(1.15,8.26)$ & 0.027 & 3.27 & $(1.20,10.05)$ \\
\hline Question 15 "lightheaded after standing for some time" & 0.168 & 0.994 & - & - & 0.996 & - & - \\
\hline Question 16 "fainted in the past 6 months" & 0.733 & 0.863 & 0.78 & $(0.03,20.15)$ & 0.815 & 1.49 & $(0.05,111.72)$ \\
\hline Question 17 "perspire excessively during the day" & 0.404 & 0.115 & 0.52 & $(0.23,1.17)$ & 0.126 & 0.52 & $(0.23,1.19)$ \\
\hline Question 18 "perspire excessively during the night" & 0.789 & 0.957 & 0.98 & $(0.40,2.40)$ & 0.963 & 1.02 & $(0.42,2.55)$ \\
\hline Question 19 "oversensitive to bright light" & 0.490 & 0.867 & 0.90 & $(0.28,3.02)$ & 0.994 & 1.00 & $(0.29,3.57)$ \\
\hline Question 20 "have trouble tolerating cold" & 0.799 & 0.690 & 1.20 & $(0.49,3.00)$ & 0.626 & 1.25 & $(0.51,3.17)$ \\
\hline Question 21 "have trouble tolerating heat" & 0.852 & 0.498 & 0.73 & $(0.29,1.84)$ & 0.517 & 0.73 & $(0.28,1.89)$ \\
\hline Question 22 "impotent (for men)" & 0.984 & 0.251 & 0.45 & $(0.10,1.72)$ & 0.221 & 0.41 & $(0.09,1.65)$ \\
\hline Question 23 "unable to ejaculate (for men)" & 0.942 & 0.251 & 0.45 & $(0.10,1.72)$ & 0.221 & 0.41 & $(0.09,1.65)$ \\
\hline $\begin{array}{l}\text { Question 23a "taken medication for an erection disorder (for } \\
\text { men)" }\end{array}$ & 1.000 & 1.000 & - & - & 1.000 & - & - \\
\hline Question 24 "vagina too dry during sexual activity (for women)" & 0.463 & 0.163 & 2.81 & $(0.71,14.15)$ & 0.177 & 2.77 & $(0.68,14.35)$ \\
\hline Question 25 "difficulty reaching an orgasm (for women)" & 0.463 & 0.163 & 2.81 & $(0.71,14.15)$ & 0.177 & 2.77 & $(0.68,14.35)$ \\
\hline Question 26a "use medication for constipation" & 0.310 & 0.313 & 1.65 & $(0.64,4.51)$ & 0.501 & 1.42 & $(0.52,4.06)$ \\
\hline Question 26b "use medication for urinary problems" & 0.381 & 0.293 & 3.31 & $(0.47,66.00)$ & 0.261 & 3.62 & $(0.50,72.98)$ \\
\hline Question 26c "use medication for blood pressure" & 0.867 & 0.867 & 0.90 & $(0.28,3.02)$ & 0.877 & 0.91 & $(0.28,3.08)$ \\
\hline Question 26d "use medication for other symptoms" & 1.000 & 1.000 & - & - & 1.000 & - & - \\
\hline
\end{tabular}

CI confidence interval, OR odds ratio, SCOPA-AUT Scales for Outcomes in Parkinson's Disease-Autonomic questionnaire, SS-16 Sniffin' Sticks 16 items

a $p$ value of the linear correlation between the score of each question and SS-16 scores

$\mathrm{b}$ the association between each symptom described by each question and olfactory dysfunction using binomial logistic regression

${ }^{c}$ adjust disease duration, gender and age

using MIBG uptake [23]. As for urinary problems, there was no evidence indicated that hyposmia was associated with that.

The association between olfactory dysfunction and constipation and urinary dysfunctions revealed that there was maybe more global involvement in non-motor symptoms in PD. Environmental factors, such as microbiological changes, may influence PD non-motor symptoms and pathogenesis of $\mathrm{PD}$ given the anatomical position of organs with olfactory function, constipation and urinary functions. More studies to discover the pathogenesis of environmental factors to PD are warranted.

The strengths of our study are that the diagnosis was based on MDS criteria. We assessed autonomic symptoms with structured scale which is widely accepted.

This study has some weakness and limitations. First, we did not perform objective clinical methods such as electrophysiology to assess autonomic symptoms. Second, we 
did not take relative medical history of several autonomic dysfunctions that SCOPA-AUT is not covered, such as diarrhea, dry eyes and dry mouth. These items were presented in Composite Autonomic Symptom Score (COMPASS) scale. Third, the sample of our study is small and our study was a single center study. More multicenter and larger studies are warranted.

\section{Conclusion}

In conclusion, gastrointestinal, urinary and a part of cardiovascular symptoms of SCOPA-AUT were associated with olfactory dysfunction in PD patients. Larger and multicentral studies are warranted.

\section{Abbreviations \\ Cl: Confidence interval; COMPASS: Composite Autonomic Symptom Score; DAT: Dopamine transporter; MDS: Movement disorders society; MIBG: 123I- metaiodobenzylguanidine; OR: Odds ratio; PD: Parkinson's disease; SCOPA- AUT: Scales for Outcomes in Parkinson's Disease-Autonomic questionnaire; SPECT: Single-photon emission computed tomography; SS-16: Sniffin' Sticks 16 \\ Funding \\ This work was supported by the National Natural Science Foundation of China [grant numbers 81601010, 81701039] and the Ministry of Science and Technology (Grant No. 2016YFA0101301), Shanghai Science and Technology Commission (Grant No. 16511105000-16511105002), Key Disciplines Group Construction Project of Pudong Health Bureau of Shanghai (Grant No. PWZxq2017-08).}

\section{Availability of data and materials}

The datasets used and/or analyzed during the current study are available from the corresponding author on reasonable request.

\section{Authors' contributions}

XYW performed the experiments of Molecular Cell Biology and data analysis; YYH supervised the morphological analysis and performed the biochemistry experiments; BZ initiated and promoted the progress of the project; GL and BZ designed experiments and wrote the manuscript. All authors read and approved the final manuscript.

\section{Ethics approval and consent to participate}

This study is approved by the Ethics Committee of East Hospital, affiliated to Shanghai Tongji University School of Medicine, Shanghai, China and all participants gave written informed consent for participation and to publication.

\section{Consent for publication}

Not applicable.

\section{Competing interests}

The authors declare that they have no competing interests.

\section{Publisher's Note}

Springer Nature remains neutral with regard to jurisdictional claims in published maps and institutional affiliations.

Received: 9 September 2018 Accepted: 28 January 2019

Published online: 02 February 2019

\section{References}

1. Kalia LV, Lang AE. Parkinson's disease. Lancet (London, England). 2015; 386(9996):896-912

2. Antony PM, Diederich NJ, Kruger R, Balling R. The hallmarks of Parkinson's disease. FEBS J. 2013;280(23):5981-93.

3. Todorova A, Jenner P, Ray Chaudhuri K. Non-motor Parkinson's: integral to motor Parkinson's, yet often neglected. Pract Neurol. 2014;14(5):310-22.
4. Doty RL. Olfactory dysfunction in Parkinson disease. Nat Rev Neurol. 2012; 8(6):329-39.

5. Morley JF, Duda JE. Olfaction as a biomarker in Parkinson's disease. Biomark Med. 2010:4(5):661-70.

6. Berg D, Postuma RB, Adler CH, Bloem BR, Chan P, Dubois B, et al. MDS research criteria for prodromal Parkinson's disease. Mov Disord. 2015;30(12):1600-11.

7. Li K, Reichmann H, Ziemssen T. Recognition and treatment of autonomic disturbances in Parkinson's disease. Expert Rev Neurother. 2015;15(10):1189-203.

8. Chen W, Xu ZM, Wang G, Chen SD. Non-motor symptoms of Parkinson's disease in China: a review of the literature. Parkinsonism Relat Disord. 2012; 18(5):446-52.

9. Jain S, Goldstein DS. Cardiovascular dysautonomia in Parkinson disease: from pathophysiology to pathogenesis. Neurobiol Dis. 2012:46(3):572-80.

10. Postuma RB, Berg D, Stern M, Poewe W, Olanow CW, Oertel W, et al. MDS clinical diagnostic criteria for Parkinson's disease. Mov Disord. 2015;30(12):1591-601.

11. Hummel T, Sekinger B, Wolf SR, Pauli E, Kobal G. Sniffin' sticks': olfactory performance assessed by the combined testing of odor identification, odor discrimination and olfactory threshold. Chem Senses. 1997;22(1):39-52.

12. Yang L, Wei Y, Yu D, Zhang J, Liu Y. Olfactory and gustatory function in healthy adult Chinese subjects. Otolaryngol Head Neck Surg. 2010;143(4):554-60.

13. Visser M, Marinus J, Stiggelbout AM, Van Hilten JJ. Assessment of autonomic dysfunction in Parkinson's disease: the SCOPA-AUT. Mov Disord. 2004;19(11): 1306-12.

14. Wu L, Mu N, Yang F, Zang J, Zheng JP. A study of the non-motor symptoms in early Parkinson's disease with olfactory deficits. Eur Rev Med Pharmacol Sci. 2016;20(18):3857-62

15. Kang P, Kloke J, Jain S. Olfactory dysfunction and parasympathetic dysautonomia in Parkinson's disease. Clin Auton Res. 2012;22(4):161-6.

16. Siderowf A, Jennings D, Eberly S, Oakes D, Hawkins KA, Ascherio A, et al. Impaired olfaction and other prodromal features in the Parkinson at-risk syndrome study. Mov Disord. 2012;27(3):406-12.

17. Ramjit AL, Sedig L, Leibner J, Wu SS, Dai Y, Okun MS, et al. The relationship between anosmia, constipation, and orthostasis and Parkinson's disease duration: results of a pilot study. Int J Neurosci. 2010;120(1):67-70.

18. Heintz-Buschart A, Pandey U, Wicke T, Sixel-Doring F, Janzen A, Sittig-Wiegand $E$, et al. The nasal and gut microbiome in Parkinson's disease and idiopathic rapid eye movement sleep behavior disorder. Mov Disord. 2018:33(1):88-98.

19. Pagano G, Yousaf T, Wilson H, Niccolini F, Polychronis S, Chaudhuri KR, et al. Constipation is not associated with dopamine transporter pathology in early drug-naive patients with Parkinson's disease. Eur J Neurol. 2018;25(2):307-12.

20. Ponsen MM, Stoffers D, Booij J, van Eck-Smit BLF, Wolters EC, Berendse HW. Idiopathic hyposmia as a preclinical sign of Parkinson's disease. Ann Neurol. 2004;56(2):173-81.

21. Bohnen NI, Gedela S, Herath P, Constantine GM, Moore RY. Selective hyposmia in Parkinson disease: association with hippocampal dopamine activity. Neurosci Lett. 2008:447(1):12-6.

22. Mizutani $Y$, Nakamura T, Okada A, Suzuki J, Watanabe H, Hirayama M, et al. Hyposmia and cardiovascular dysautonomia correlatively appear in earlystage Parkinson's disease. Parkinsonism Relat Disord. 2014;20(5):520-4.

23. lijima M, Osawa M, Momose M, Kobayakawa T, Saito S, Iwata M, et al. Cardiac sympathetic degeneration correlates with olfactory function in Parkinson's disease. Mov Disord. 2010;25(9):1143-9.

Ready to submit your research? Choose BMC and benefit from:

- fast, convenient online submission

- thorough peer review by experienced researchers in your field

- rapid publication on acceptance

- support for research data, including large and complex data types

- gold Open Access which fosters wider collaboration and increased citations

- maximum visibility for your research: over $100 \mathrm{M}$ website views per year

At BMC, research is always in progress.

Learn more biomedcentral.com/submissions 